\title{
Reflets
}

Revue ontaroise d'intervention sociale et communautaire

\section{Population vieillissante oblige : formation à l'intention des intervenants en santé et services sociaux}

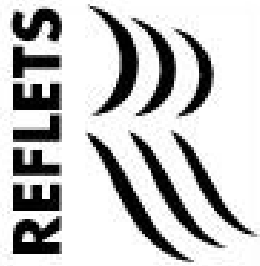

\section{Rachel Ouellette et Roland Lecomte}

Volume 8, numéro 1, printemps 2002

La réadaptation : son visage français en Ontario

URI : https://id.erudit.org/iderudit/026380ar

DOI : https://doi.org/10.7202/026380ar

Aller au sommaire du numéro

Éditeur(s)

Reflets : Revue ontaroise d'intervention sociale et communautaire

ISSN

1203-4576 (imprimé)

1712-8498 (numérique)

Découvrir la revue

Citer cet article

Ouellette, R. \& Lecomte, R. (2002). Population vieillissante oblige : formation à l'intention des intervenants en santé et services sociaux. Reflets, 8(1), 158-163. https://doi.org/10.7202/026380ar

Tous droits réservés (C) Reflets : Revue ontaroise d'intervention sociale et communautaire, 2002
Ce document est protégé par la loi sur le droit d'auteur. L'utilisation des services d'Érudit (y compris la reproduction) est assujettie à sa politique d'utilisation que vous pouvez consulter en ligne.

https://apropos.erudit.org/fr/usagers/politique-dutilisation/ 


\title{
Population vieillissante oblige : formation à l'intention des intervenants en santé et services sociaux
}

\author{
Rachel Ouellette \\ Centre national de formation en santé \\ Roland Lecomte \\ Programme de gérontologie, Université d'Ottawa
}

\section{Une population changeante et diversifiée}

Le vieillissement de la population et l'accroissement de la population aînée sont des phénomènes marquants de notre époque. On les associe principalement à l'augmentation de l'espérance de vie et à la baisse du taux de natalité. Touchant le Canada comme la plupart des pays occidentaux, ces phénomènes ne sont pas sans conséquences pour l'organisation sociale, politique, et économique mais aussi pour les systèmes de santé (Haentjens et al. 1999). Le présent article propose un survol des considérations relatives à cette population changeante et diversifiée, ayant mené à la création d'un nouveau programme de formation en gérontologie.

On associe généralement à la catégorie âgée ou aînée les personnes de 65 ans et plus. Cette définition s'inspire de l'âge traditionnel de la retraite qui demeure officiellement fixé à 65 ans. Le dernier recensement de Statistique Canada (1996) a permis 
de dénombrer 3,6 millions de personnes âgées de 65 ans et plus au pays, soit près de $12 \%$ de la population totale. Au cours des 15 dernières années, c'est ce segment de la population qui a connu la croissance la plus rapide. L'importance de la population âgée connait quelques variations d'une région à l'autre du pays. En Ontario, la proportion de personnes âgées rejoint la moyenne nationale se situant à $11,7 \%$ de la population totale. Du côté de la population francophone, on dénombre près de 68000 personnes de 65 ans et plus en Ontario, ce qui représente $13 \%$ de cette population.

En même temps qu'elle s'accroît, la population âgée voit son visage se transformer (Haentjens et al. 1999). Si d'un côté elle vieillit, avec le prolongement de la longévité, elle tend pourtant à s'éloigner de cette image préconçue qu'on se faisait d'elle il n'y a pas si longtemps. En effet, l'image négative souvent caractérisée par la maladie, l'inactivité et la prise en charge par la société ne colle pas à la population âgée d'aujourd'hui qui s'avère de plus en plus hétérogène (Lesemann 2000). Dans le contexte des départs à la retraite qui sont davantage précoces au sein de certaines strates de la population, on a maintenant tendance à inclure dans la catégorie âgée les personnes de 60 ans et plus, de 55 ans et plus et même à partir de 50 ans. On assiste donc à une subdivision en plusieurs segments de la population aînée. L'apparition de l'expression quatrième âge vient souligner cette subdivision en marquant la différence qui existe entre les aînés plus âgés et les moins âgés (Haentjens et al. 1999). Cette diversité s'exprime d'ailleurs par l'étendue et la variété des besoins de cette population élargie et changeante et ce, tant sur le plan social, économique que de la santé.

Le phénomène du vieillissement et l'état de santé de la population âgée ont longtemps été l'objet de mythes et de stéréotypes (Kelchner 1999).Vieillissement et problèmes de santé ont en effet souvent été considérés comme des inséparables. Tout en identifiant qu'une partie de la population âgée est sujette à des problèmes de santé graves ou chroniques, les statistiques sur l'état de santé des Canadiens démontrent toutefois que la majorité des aînés présente une condition physique satisfaisante et continue 
de mener une vie active et autonome (Haentjens et al. 1999). Par contre, en avançant en âge, ces personnes voient augmenter leurs risques de maladie. L'incidence des problèmes grandit en effet chez les 75 ans et plus, cohorte qui va en s'accroissant. Les besoins en termes de soins de santé different donc considérablement entre les segments de la population âgée (Clarke et al., 2000).

C'est peut-être sur le plan socio-économique que la situation des personnes âgées s'apparente le plus à l'idée traditionnelle qu'on s'en fait. Bien qu'il faille là encore éviter de généraliser, il est reconnu que la population aînée représente souvent un groupe socio-économiquement défavorisé. La situation est généralement pire au niveau de la population aînée francophone. Cela représente un facteur de risque additionnel lié à la santé des aînés. Encore faut-il souligner ici les disparités observées entre les jeunes aînés et les aînés plus âgés (Brotman 1998), de même que celles entre les hommes et les femmes (Stuckelberger et al.1996) et de celles entre les groupes ethniques (Arcand 1982).

\section{Besoin de nouvelles compétences}

La part croissante qu'occupe la population aînée par rapport à la population totale a des répercussions certaines sur les rouages de notre société, notamment sur l'organisation et la planification de certains services publics. Dans le secteur de la santé et des services sociaux, l'évolution et la diversité des besoins des aînés, combinées aux réalités du contexte économique, ont engendré des changements importants en matière de soins aux aînés, par exemple sur les plans du maintien à domicile, de la désinstitutionnalisation ainsi que des concepts de promotion de la santé et de prévention de la maladie.

Ces transformations se répercutent aussi sur les besoins en matière de formation de la main-d'œuvre dans ce secteur. Bien que le vieillissement de la population ne soit pas un phénomène nouveau en soi, la prise de conscience grandissante relativement 
aux besoins en émergence de ce segment diversifié de la population mène à mieux comprendre les compétences particulières qu'il exige. En effet, l'acquisition de connaissances relatives à cette clientèle n'est plus considérée comme un atout mais plutôt comme une nécessité si l'on veut s'assurer de répondre adéquatement à ses besoins et de posséder tous les outils requis pour ce faire (Lesemann 2001). Il appert effectivement essentiel de mieux comprendre ce qu'est le vieillissement, ce que sont les réalités et les changements auxquels font face les aînés ainsi que la part entière qu'ils occupent au sein de notre société et ce, afin de mieux les servir et d'éliminer les stéréotypes à leur égard. Des connaissances au sujet des aspects physiologiques, pathologiques, psychologiques, sociaux ou économiques du vieillissement, pour ne nommer que ceux-là doivent faire partie du savoir de base des intervenants de la santé et des services sociaux appelés à œuvrer auprès de la clientèle âgée. Les professionnels des disciplines de la réadaptation font, bien entendu, partie de ces intervenants clés auxquels nous faisons ici référence.

Suite à l'identification de ce besoin de formation complémentaire au sein de différentes disciplines dans le domaine de la santé et des services sociaux mais aussi au sein de nombreuses autres professions d'aide, une vingtaine de programmes de formation en gérontologie ont été développés dans les universités canadiennes au cours des quinze dernières années, dont dix en Ontario. Pour la majorité de ceux-ci, il s'agit de programmes de certificat. Des programmes ont également été développés au niveau collégial. Jusqu'à tout récemment, aucun des programmes de formation universitaire en gérontologie offerts en Ontario n'était disponible en français.Toutefois, depuis septembre 2001, l'Université d'Ottawa offre un programme de gérontologie disponible entièrement en français.

Ce programme a été conçu à partir des besoins recueillis auprès d'étudiants, d'employeurs et d'intervenants du milieu de la santé et des services sociaux de l'Est de l'Ontario (Haentjens et al. 1999). Les besoins en matière de formation furent exprimés autour de trois axes : fournir des explications scientifiques au vieillissement individuel et au vieillissement des populations; identifier des 
interventions pertinentes autant au niveau des individus et de la communauté qu'en matière de politiques sociales et, finalement, proposer des méthodes de recherche appropriées. Il fut convenu dès le départ que le programme de formation se devait d'exposer les étudiants aux problématiques du vieillissement et de la santé par le biais d'une perspective interdisciplinaire et de favoriser le développement et l'intégration comme champ d'étude des connaissances théoriques, pratiques et de recherche en gérontologie.

Afin de répondre aux besoins identifiés, le programme offre trois options. D'abord, pour combler le besoin de spécialisation des étudiants inscrits dans différents programmes en sciences sociales, aux arts et en sciences de la santé, le programme offre une double concentration, semblable à une mineure, en gérontologie. De plus, un certificat est aussi offert aux intervenants déjà sur le marché du travail qui désirent parfaire leurs connaissances en gérontologie. Enfin, grâce à un partenariat avec La Cité collégiale, le programme permet d'ajouter un volet pratique à la formation par la participation à des stages cliniques en milieux d'intervention. Afin de favoriser un accès accru à ce programme de formation, en Ontario et ailleurs au pays, plusieurs des cours sont disponibles à distance via la vidéo-conférence et ce, grâce à la collaboration du Centre national de formation en santé. Ce programme est sous l'égide de la Faculté des sciences sociales et pour en obtenir tous les détails il suffit de visiter le site internet suivant : www.sciencessociales.uottawa.ca.

\section{Somme toute...}

On parle de vieillissement de la population depuis plusieurs années déjà. Les transformations engagées par notre société pour y répondre sont toutefois encore bien modestes. Elles ne reflètent que timidement l'ampleur des bouleversements démographiques présents et à venir. L'arrivée prochaine des baby-boomers à l'âge 
vénérable de la retraite est considérée par plusieurs comme un élément central qui agira comme agent catalyseur et accélérateur de changements (Reinhardt 2000; Wagner 2000). Il est clair que les besoins des personnes âgées et les demandes de service pour y répondre, dans différents secteurs d'activités, iront en augmentant.

Dans ce contexte, l'acquisition de connaissances étendues liées à cette population particulière ne peut que représenter un atout important pour ne pas dire déterminant pour les différents intervenants de la santé et des services sociaux qui auront vraisemblablement à travailler avec cette clientèle à un moment ou à un autre. Qu'on y pense en termes de formation initiale ou de formation continue, l'acquisition de connaissances liées à la population aînée nous servira éventuellement à tous. Après tout, comme l'exprime si bien Carlson «Vieillir, c'est encore le seul moyen que l'on ait trouvé pour vivre longtemps ».

\section{Références}

ARCAND, B. (1982). "La construction culturelle de la vieillesse ", Anthropologies et sociétés, 3, 7-23. BROTMAN, S. (1998). "The Incidence of Poverty among Seniors in Canada : Exploring the Impact of Gender, Ethnicity and Race », Canadian Journal of Aging, 17:2, 166-185.

CLARKE, P., MARSHALL, V., RYFF, C., et ROSENTHAL, C. (2000). «Well-Being in Canadian Seniors : Findings from the Canadian Study of Health and Aging ", Canadian Journal on Aging, 19:2, 139-159.

HAENTIENS, M., et LEMIRE, D. (1999). Évaluation de la pertinence et de la faisabilité d'un programme de formation en gérontologie pour la population francophone de l'Ontario. Recherche parrainée par l'Université d'Ottawa sous la direction de Roland Lecomte.

KELCHNER, E.S. (1999). «Ageism's Impact and Effect on Society : Not just a concern for the Old ", Journal of Gerontological Social Work, 32:4, 85-100.

LESEMANN, F. (2000). "Les nouveaux visages du vieillissement », Le Gérontophile, 22:3, 9-12.

LESEMANN, F. (2001). «Twenty Years of Canadian Research on Aging : an attempted understanding ", Canadian Journal on Aging, 20(Suppl.1), 58-66.

REINHARDT, U.E. (2000). "Health Care for the Aging Baby-boom : lessons from abroad ", Journal of Economic Perspectives, 14:2, 71-83.

STATISTIQUE CANADA (1996). Données tirée du recensement de 1996.

STUCKELBER GER,A., \& F. HÖOFLINGER, F. (1996). Vieillissement différentiel : hommes et femmes, Zürich: Éditions Seismo, 192.

WAGNER, L., (2000). "Meeting the Baby Boomer Challenge », Provider, Jan. 2000, 26:1, 29-36. 